https://helda.helsinki.fi

\title{
Proteomics to study macrophage response to viral infection
}

\section{Nyman, Tuula A.}

2018-05-30

Nyman , T A \& Matikainen , S 2018 , ' Proteomics to study macrophage response to viral infection ' , Journal of Proteomics, vol. 180 , pp. 99-107 . https://doi.org/10.1016/j.jprot.2017.06.018

http://hdl.handle.net/10138/302416

https://doi.org/10.1016/j.jprot.2017.06.018

publishedVersion

Downloaded from Helda, University of Helsinki institutional repository.

This is an electronic reprint of the original article.

This reprint may differ from the original in pagination and typographic detail.

Please cite the original version. 


\title{
Review
}

\section{Proteomics to study macrophage response to viral infection}

\author{
Tuula A. Nyman ${ }^{\mathrm{a}, *}$, Sampsa Matikainen ${ }^{\mathrm{b}}$ \\ a Department of Immunology, Institute of Clinical Medicine, University of Oslo and Rikshospitalet Oslo, Oslo, Norway \\ ${ }^{\mathrm{b}}$ University of Helsinki and Helsinki University Hospital, Rheumatology, Helsinki, Finland
}

\section{A R T I C L E I N F O}

\section{Article history:}

Received 31 March 2017

Received in revised form 12 June 2017

Accepted 19 June 2017

Available online 21 June 2017

\section{Keywords:}

Proteomics

Macrophage

Viral infection

Innate immunity

\begin{abstract}
A B S T R A C T
Viral infections are a major burden to human and animal health. Immune response against viruses consists of innate and adaptive immunity which are both critical for the eradication of the viral infection. The innate immune system is the first line of defense against viral infections. Proper innate immune response is required for the activation of adaptive, humoral and cell-mediated immunity. Macrophages are innate immune cells which have a central role in detecting viral infections including influenza A and human immunodeficiency viruses. Macrophages and other host cells respond to viral infection by modulating their protein expression levels, proteins' posttranslational modifications, as well as proteins' intracellular localization and secretion. Therefore the detailed characterization how viruses dynamically manipulate host proteome is needed for understanding the molecular mechanisms of viral infection. It is critical to identify cellular host factors which are exploited by different viruses, and which are less prone for mutations and could serve as potential targets for novel antiviral compounds. Here, we review how proteomics studies have enhanced our understanding of macrophage response to viral infection with special focus on Influenza A and Human immunodeficiency viruses, and virus infections of swine.

Significance: Influenza A viruses (IAVs) and human immunodeficiency viruses (HIV) infect annually millions of people worldwide and they form a severe threat to human health. Both IAVs and HIV-1 can efficiently antagonize host response and develop drug-resistant variants. Most current antiviral drugs are directed against viral proteins, and there is a constant need to develop new next-generation drugs targeting host proteins that are essential for viral replication. Porcine reproductive and respiratory syndrome virus (PRRSV) and porcine circovirus type 2 (PCV2) are economically important swine pathogens. Both PRRSV and PCV2 cause severe respiratory tract illnesses in swine. IAVs, HIV-1, and swine viruses infect macrophages activating antiviral response against these viruses. Macrophages also have a central role in the replication and spread of these viruses. However, macrophage response to these viruses is incompletely understood. Current proteomics methods can provide a global view of host-response to viral infection which is needed for in-depth understanding the molecular mechanisms of viral infection. Here we review the current proteomics studies on macrophage response to viral infection and provide insight into the global host proteome changes upon viral infection.
\end{abstract}

(c) 2017 Elsevier B.V. All rights reserved.

\section{Contents}

1. Macrophages have a central role in the antiviral response against human and swine pathogenic viruses. . . . . . . . . . . . . . . . . 100

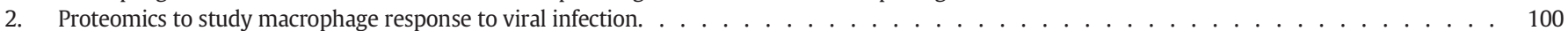

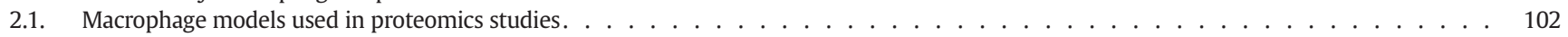

2.2. Two-dimensional electrophoresis based proteome studies of IAV and HIV infected macrophages. . . . . . . . . . . . . . . . . . . 103

2.3. MS-based proteomics studies of macrophage response to influenza A virus infection . . . . . . . . . . . . . . . . . . . . . 103

2.4. MS-based proteomics to characterize HIV-1 infection in human macrophages . . . . . . . . . . . . . . . . . . . . . . . . 104

2.5. Proteomics to study macrophage response to porcine virus infection . . . . . . . . . . . . . . . . . . . . . . . . . . . . . . . . . . . .

3. Global protein secretion studies of virally infected macrophages. . . . . . . . . . . . . . . . . . . . . . . . . . 104

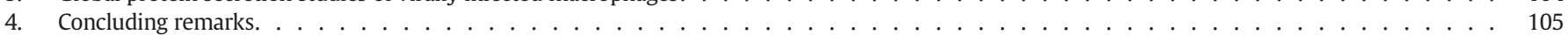

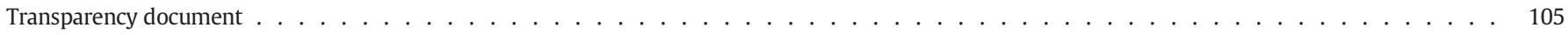

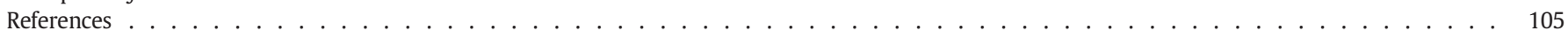

* Corresponding author at: Department of Immunology, Faculty of Medicine, University of Oslo, Sognsvannsveien 20, Rikshospitalet, 0372 Oslo, Norway.

E-mail address: tuula.nyman@medisin.uio.no (T.A. Nyman). 


\section{Macrophages have a central role in the antiviral response against human and swine pathogenic viruses}

Macrophages are innate immune cells which have a central role in detecting viral infections including influenza $A$ and human immunodeficiency viruses. They are found in all parts of the body, and they constitute $5-15 \%$ of cells depending on tissue. They show great functional diversity and in addition to activating innate immune response they have central roles in development, homeostasis, and tissue repair. Activation of macrophages by viral infection results in antiviral response and inflammation aiming to pathogen elimination. Upon activation, macrophages start to secrete cytokines to mount inflammatory response, chemokines to recruit other immune cells to the site of infection or inflammation, and other proteins inducing antiviral defense and tissue regeneration.

Influenza A viruses (IAVs) and human immunodeficiency viruses (HIV) infect annually millions of people worldwide and they form a severe threat to human health. Both viruses have single-stranded RNA genomes. IAVs have negative-stranded RNA genome and they replicate through positive-stranded RNA whereas HIVs replicate through a DNA intermediate to produce viral RNA. IAVs belong to Orthomyxoviridae family that are capable of infecting humans, birds, and swine. They are respiratory pathogens that spread around the world in seasonal epidemics, resulting in the deaths of hundreds of thousands worldwide annually, and millions in pandemic years (http://who.int/topics/ influenza/en/).

Infection with HIV is pandemic with $>30$ million people estimated to be infected worldwide (http://www.who.int/hiv/en/). HIV is a human retrovirus that targets cells within the immune system and establishes lifelong infection resulting in immunodeficiency and the development of AIDS. Several drugs against HIV-1 and IAV are available and many are in development. Both IAVs and HIV-1 can efficiently antagonize host response and develop drug-resistant variants. Most current antiviral drugs are directed against viral proteins, and there is a constant need to develop new next-generation drugs targeting host proteins that are essential for viral replication.

The primary targets of IAVs are the epithelial cells of the upper respiratory tract. IAVs also infect alveolar macrophages that have a central role in controlling the spread of IAVs. Both macrophages and CD4-positive $T$ cells are important target cells for the Human Immunodeficiency Virus Type I (HIV-1). Macrophages are much more resistant to cytopathic effects of lentiviral replication than CD4-positive T cells. HIV-infected macrophages represent a stable viral reservoir with continuous virus production contributing to the pathogenesis of HIV-1 infection. It has been suggested that interaction of HIV-1 with macrophages is crucial at all stages of HIV-1 infection [1].

In addition to IAVs, porcine reproductive and respiratory syndrome virus (PRRSV) and porcine circovirus type 2 (PCV2) are economically important swine pathogens [2]. PRRSV and PCV2 cause respiratory tract illness and postweaning multisystemic wasting syndrome in swines, respectively. Both of these viruses replicate in macrophages and this virus-macrophage interaction essentially contributes to the pathogenesis of these porcine viruses. However, the macrophage response to these swine viruses is incompletely understood.

\section{Proteomics to study macrophage response to viral infection}

Viruses have coevolved with their hosts, and have multiple ways of to capture and manipulate host cellular processes for their replication and spread. Macrophages and other host cells respond to viral infection by modulating their protein expression levels, proteins' posttranslational modifications, as well as proteins' intracellular localization and secretion. Therefore detailed characterization how viruses dynamically manipulate host proteome is needed for understanding the molecular mechanisms of viral infection. Current proteomics methods can provide a global view of host-response to viral infection, both related to host cell

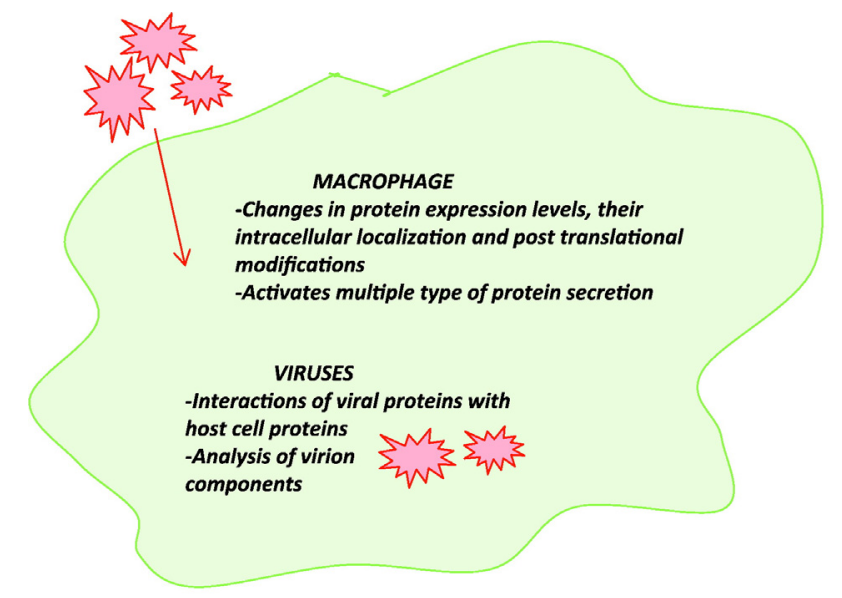

Fig. 1. Viruses have multiple ways of to capture and manipulate host cellular processes for their replication and spread. Viral infection can modulate host cell proteome at multiple levels. Viral proteins also interact with host cells proteins, and proteomics provides tools to characterize these interactions.

response to virus and how viral proteins interact with host proteins (Fig. 1).

In proteomics, the first 'workhorse' technique was two-dimensional electrophoresis (2-DE) combined with protein identification by mass spectrometry (MS). This combination was by far the most commonly used proteomics platform in 1990's and early 2000's. The main advantage of 2-DE is that it has excellent separation efficiency for intact proteins, and even protein isoforms can be separated into distinct spots. Also post-translationally modified or truncated/fragmented proteins will be separated from non-modified/intact proteins. However, 2-DE requires a lot of manual lab work and the reproducibility of the gels is highly dependent on the skills of the person running the gels. Also the quantification accuracy depends on the staining method used and comparison analysis of the gels can be time-consuming. The developments of high-resolution MS, optimized sample preparation protocols and advanced data-analysis tools have made proteomics very MS-centric during the last decade. This can also be clearly seen with proteomics studies on viral infection where majority of the new publications employ various MS-based proteomics workflows. A powerful example of this is a recent study by Weekes et al. who developed a MS-based proteomic technique called "quantitative temporal viromics" [3] and used it to characterize Human cytomegalovirus (HCMV) infection in primary human fetal foreskin fibroblasts. They quantified $>8000$ proteins from fibroblasts including $>1000$ cell-surface proteins and $81 \%$ of all canonical HCMV proteins over eight time points during productive infection, providing a near-complete temporal view of the host proteome and HCMV virome.

Macrophages responses to IAV and HIV have been extensively studied using different proteomics workflows including 2-DE and MS-based methods (Tables 1 and 2). Different proteomic methods have also been used to study pulmonary alveolar macrophages following porcine reproductive and respiratory syndrome virus (PRRSV) and porcine circovirus type 2 infection (Table 3 ). The proteomic methods used in different studies are summarized in Fig. 2. With HIV, earliest proteomics reports were using SELDI-TOF Protein Chips [4-10] which is a variant of MALDI analysis. SELDI-TOF combines surface-enhanced selective capture of proteins with their time-of-flight separation and detection, however SELDI alone is not capable of identifying proteins from the samples and is therefore of limited use anymore. These studies identified several proteins that were differentially expressed in sera from HIV-infected individuals with or without HIV-1-associated dementia [11].

In host-virus protein interaction studies, different affinity purification (AP) methods are combined with MS-based protein identification 
Table 1

Proteomic analysis of macrophage response to influenza A virus infection and proteomic studies of influenza viruses.

\begin{tabular}{|c|c|c|c|}
\hline Reference & IAV strain & Host cell & Proteomic method(s) \\
\hline Öhman 2009 [19] & Udorn/307/1972 (H3N2) & $\begin{array}{l}\text { Human monocyte-derived } \\
\text { macrophages }\end{array}$ & Subcellular proteomics; 2-DE and MS \\
\hline Lietzen 2011 [25] & Udorn/307/1972 (H3N2)* & $\begin{array}{l}\text { Human monocyte-derived } \\
\text { macrophages }\end{array}$ & $\begin{array}{l}\text { Subcellular proteomics and secretomics combined with kinetic analysis; iTRAQ } \\
\text { and LC-MS/MS }\end{array}$ \\
\hline Zhu 2012 [20] & $\begin{array}{l}\text { F9/10 and 34/06 swine viruses } \\
\text { (H1N1) }\end{array}$ & Porcine alveolar macrophages & Kinetic analysis of total cellular lysates; 2-DE and MS \\
\hline Liu 2012 [16] & $\mathrm{PR} / 8 / 34(\mathrm{H} 1 \mathrm{~N} 1)$ & Human alveolar macrophages & Total cellular lysates, SILAC \\
\hline Cheung 2012 [24] & Vietnam/3212/04 (H5N1) & $\begin{array}{l}\text { Human monocyte-derived } \\
\text { macrophages }\end{array}$ & Kinetic analysis of total cellular lysates, label-free quantification \\
\hline $\begin{array}{l}\text { Söderholm } 2016 \\
\text { [31] }\end{array}$ & Udorn/307/1972 (H3N2) & $\begin{array}{l}\text { Human monocyte-derived } \\
\text { macrophages }\end{array}$ & Phosphoproteomics (IMAC and LC-MS/MS) combined with functional studies \\
\hline \multirow[t]{2}{*}{ Cypryk 2016 [48] } & Udorn/307/1972 (H3N2) & $\begin{array}{l}\text { Human monocyte-derived } \\
\text { macrophages }\end{array}$ & Extracellular vesicle analysis by LC-MS/MS \\
\hline & *Also Beijing/353/89 & & \\
\hline \multicolumn{3}{|l|}{ Reference } & Proteomics of influenza virus proteins \\
\hline \multirow{2}{*}{\multicolumn{3}{|c|}{$\begin{array}{l}\text { Hutchinson } 2012 \text { [32] } \\
\text { Hutchinson } 2014 \text { [57] }\end{array}$}} & Mapping the phosphoproteome of influenza A and B viruses by mass spectrometry \\
\hline & & & Conserved and host-specific features of influenza virion architecture \\
\hline
\end{tabular}

and quantification. This has been a very active field of research in proteomics applied to virology for most host cell types and viral infections [12]. As an example, Heaton et al. [13] used AP-MS to generate an interactome map of host and IAV proteins during an active infection in human lung epithelial cell line A549. They generated fully infectious tagged influenza viruses and used infection-based proteomics to identify pivotal arms of cellular signaling required for influenza virus growth and infectivity. However, with macrophages very little has been done

Table 2

Proteomic studies of macrophages upon HIV infection and proteome analysis of HIV virions.

\begin{tabular}{|c|c|}
\hline Reference & SELDI-TOF protein chip \\
\hline Luo 2003 [4] & Macrophage proteomic fingerprinting predicting HIV-1-associated cognitive impairment \\
\hline Carlson 2004 [5] & A preliminary report for proteomic fingerprinting of HIV-1-infected human MDMs \\
\hline Wojna 2004 [6] & Proteomic fingerprinting of human immunodeficiency virus type 1-associated dementia (a case study) \\
\hline Kadiu 2007 [7] & Secretome analyses of culture fluids derived from HIV-1 infected monocyte-derived macrophages \\
\hline $\begin{array}{l}\text { Luciano-Montalvo } 2008 \\
\text { [8] }\end{array}$ & Proteomics to identify differences between the uninfected and HIV-infected PM and MDM protein profiles linked to viral growth \\
\hline Garcia 2009 [9] & $\begin{array}{l}\text { SELDI-TOF to test the hypothesis that placental macrophages secrete different proteins as compared to MDM that may contribute to decreased HIV- } 1 \\
\text { replication }\end{array}$ \\
\hline \multirow[t]{2}{*}{ Toro-Nieves 2009 [10] } & Cell lysates of uninfected and HIV-1-infected MDMs at 14 days post infection analyzed by SELDI-TOF \\
\hline & DIGE \\
\hline $\begin{array}{l}\text { Ricardo-Dukelow } 2007 \\
\text { [70] }\end{array}$ & Proteomics to determine the effects that HIV-1-infected macrophages have on human brain microvascular endothelial cell (HBMEC) protein profiles \\
\hline Wang 2008 [23] & MP-astrocyte crosstalk was investigated by DIGE and MS analysis using vesicular stomatitis virus pseudotyped HIV infected murine microglia \\
\hline Wang 2008 [22] & Proteomics study of co-culture systems of HIV-infected mouse astrocytes and microglia \\
\hline \multirow[t]{2}{*}{ Kadiu $2011[71]$} & DIGE and MS analysis of bridging conduits from HIV infected MDMs \\
\hline & Other \\
\hline Ciborowski 2004 [72] & Analysis of conditioned serum-free medium of HIV-1ADA-infected and uninfected cells using SDS-PAGE and MS \\
\hline Ciborowski 2007 [52] & Secretome analysis of HIV-1-infected human monocyte-derived macrophages using SDS-PAGE and MS \\
\hline Brown 2008 [73] & Immunoblot analysis of about 1000 intracellular proteins from human macrophages over the course of HIV-1 infection \\
\hline \multirow[t]{2}{*}{$\begin{array}{l}\text { Luciano-Montalvo } 2009 \\
\text { [74] }\end{array}$} & $\begin{array}{l}\text { IP-studies showing that the levels of STAT-1 tyrosine phosphorylation (but not serine phosphorylation) between uninfected and HIV-infected PM } \\
\text { and MDM are differentially regulated }\end{array}$ \\
\hline & MS-based proteomics \\
\hline Pathak 2009 [75] & The effects of HIV infection on the protein profile of undifferentiated monocyte-like THP-1 cells were examined using SILAC \\
\hline Huang 2010 [76] & iTRAQ to identify differential protein expression between HIV-1-infected bone marrow-derived macrophages with and without Treg \\
\hline Kraft-Terry 2011 [34] & Alterations in de novo protein synthesis of HIV-1 infected human MDMs were examined after 3, 5, and 7 days of viral infection using SILAC \\
\hline Raposo 2011 [77] & Large-scale anti-CD4 IPs in human primary M $\varphi$ followed by high-resolution MS \\
\hline Kadiu 2012 [55] & $\begin{array}{l}\text { Cytokine arrays and GeLC-MS/MS characterization of the proteomes from unfractionated MV and exosomes (Uninfected Vesicles) and infected MV } \\
\text { and exosome proteomes }\end{array}$ \\
\hline Barrero 2013 [36] & SILAC to to characterize the Human immunodeficiency virus type 1 encoded viral protein (Vpr) response in macrophages \\
\hline Haverland 2014 [35] & Quantitative proteomics by SWATH-MS reveals altered expression of nucleic acid binding and regulatory proteins in HIV-1-infected macrophages \\
\hline Arainga 2015 [78] & SWATH-MS proteomics and cell profiling to nanoparticle atazanavir (nanoATV)-treated and HIV-1 infected human MDMs \\
\hline Du 2015 [79] & iTRAQ analysis of equine monocyte-derived macrophages infected with equine infectious anemia virus \\
\hline $\begin{array}{l}\text { Cantares-Rosario } 2015 \\
\text { [54] }\end{array}$ & Label-fee quantification and identification of macrophage-secreted cathepsin B protein interactions extracellularly \\
\hline Colon 2016 [53] & Macrophage secretome analysis using iTRAQ from women with HIV-associated neurocognitive disorders \\
\hline \multirow[t]{2}{*}{ Ganji 2016 [18] } & $\begin{array}{l}\text { Proteomics of phagosome-enriched fractions from Mycobacterium bovis Bacillus Calmette-Guérin (BCG) mono-infected and HIV-M. bovis BCG } \\
\text { co-infected THP-1 cells by LC-MALDI-MS/MS }\end{array}$ \\
\hline & Proteome analysis of HIV virions using MS-based approaches \\
\hline Chertova 2006 [80] & Proteome characterization of highly purified HIV-1 virions produced from human monocyte-derived macrophages (MDMs) \\
\hline Santos 2012 [81] & GeLC-MS/MS-based proteome analysis of HIV-1 viral cores \\
\hline Linde 2013 [58] & Determining the host protein composition in HIV virions using alternative purification technique (density modification, DM) and iTRAQ \\
\hline
\end{tabular}


Table 3

Proteomic analysis of porcine alveolar macrophages infected with PRRSV or PCV2.

\begin{tabular}{|c|c|c|}
\hline Reference & Virus & Infection time and proteomic method(s) \\
\hline $\begin{array}{l}\text { Ding } 2012 \\
\text { [37] }\end{array}$ & PRRSV & $24 \mathrm{~h}$ Infection, 2-DE analysis of total cellular lysate \\
\hline $\begin{array}{l}\text { Zhou } 2014 \\
{[38]}\end{array}$ & PRRSV & $\begin{array}{l}48 \mathrm{~h} \text { Infection, DIGE of total cellular lysate; comparison of } \\
\text { host response to highly pathogenic virus strain and } \\
\text { attenuated strain }\end{array}$ \\
\hline $\begin{array}{c}\text { Lu } 2012 \\
{[39]}\end{array}$ & PRRSV & $\begin{array}{l}\text { Kinetic study ( } 12 \mathrm{~h}, 24 \mathrm{~h}, 36 \mathrm{~h} \text { and } 48 \mathrm{~h} \text { infection) of total } \\
\text { cellular lysates; iTRAQ }\end{array}$ \\
\hline $\begin{array}{l}\text { Liu } 2013 \\
\quad[40]\end{array}$ & PCV2 & $\begin{array}{l}\text { Kinetic study ( } 12 \mathrm{~h}, 24 \mathrm{~h}, 36 \mathrm{~h} \text { and } 48 \mathrm{~h} \text { infection) of total } \\
\text { cellular lysates; iTRAQ }\end{array}$ \\
\hline $\begin{array}{l}\text { Luo } 2014 \\
\quad[41]\end{array}$ & PRRSV & $\begin{array}{l}12 \mathrm{~h} \text { and } 36 \mathrm{~h} \text { infection, phosphopeptide enrichment with } \\
\text { TiO2 and label-free quantitative phosphoproteomics }\end{array}$ \\
\hline
\end{tabular}

with AP-MS but proteomics studies have been focused on macrophages response to viral infection.

\subsection{Macrophage models used in proteomics studies}

Macrophages are heterogeneous and versatile cells found in virtually all tissues. Tissue macrophages are derived from embryonic progenitors and they renew independently of hematopoietic stem cells [14]. During infection or injury, large amounts of monocytes are recruited from the circulation and they differentiate to macrophages as they migrate into the affected tissue. Most experimental systems that study macrophage responses to virus infections use monocyte-derived macrophages (MDMs). Monocytes can be differentiated into macrophages in the presence of granulocyte-macrophage colony-stimulating factor (GMCSF) or macrophage colony-stimulating factor (M-CSF). M-CSF macrophages resemble tissue macrophages that promote healing and growth whereas GM-CSF macrophages are pro-inflammatory. GM-CSF macrophages also resemble alveolar macrophages [15] and therefore they are a good model system to study the effect of IAV infection on macrophage response. Very few studies have utilized primary human macrophages in proteomics studies, for example human primary alveolar macrophages have been used to study host response to IAV infection [16].

In proteomics studies cell lines have been widely used. The main advantage of using cell lines is that they are easy to culture and it is possible to obtain large amounts of starting material. THP-1, U-937, and HL-60 are myeloid leukemia cell lines which can be differentiated into macrophage-like adherent cells after treatment with phorbol

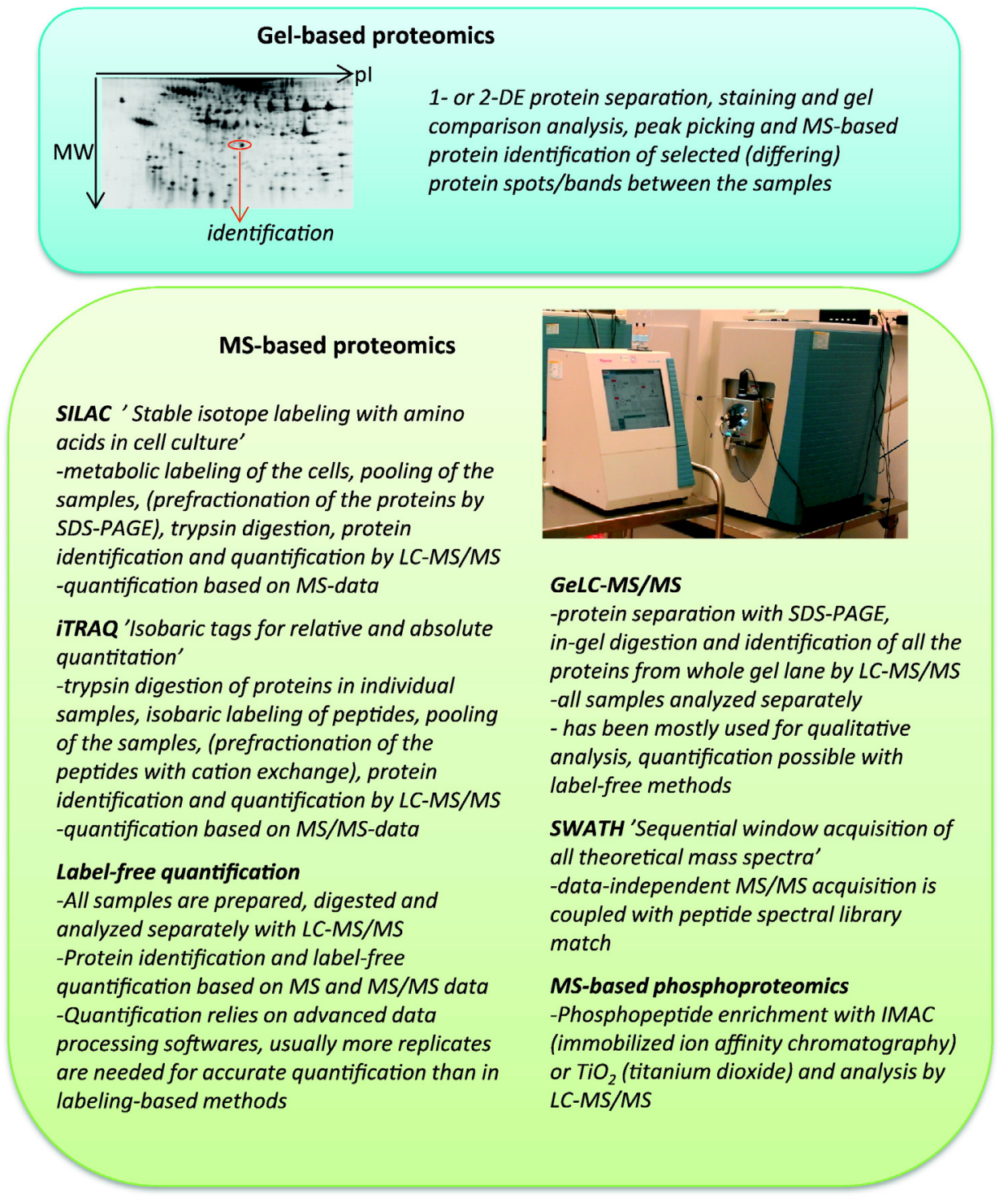

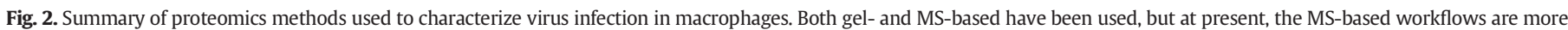
common. These different MS-based methods have been recently reviewed [67-69]. 
esters. From these, THP-1 cells are most commonly used to study macrophage biology. THP-1 cells differentiate into macrophage-like cells which mimic native monocyte-derived macrophages in some respects. However, it is important to recognize that THP-1 cells and primary monocyte-macrophages have significant differences [17]. Some proteomics studies have utilized THP-1 cells: Ganji et al. have used THP- 1 cells to study the effect of mycobacteria and HIV-1 co-infection on mycobacterial protein expression [18].

\subsection{Two-dimensional electrophoresis based proteome studies of IAV and HIV infected macrophages}

Proteome changes in macrophages upon IAV infection have been studied using 2-DE based proteomics by us and others [19,20]. Öhman et al. [19] and Zhu et al. [20] used 2-DE with silver-staining followed by in-gel digestion and MALDI-TOF/TOF analysis for protein identification to characterize influenza virus infected macrophages. Öhman et al. fractionated human MDMs into mitochondria and cytoplasm, and analyzed these fractions separately to gain sub-cellular information on protein changes [19] whereas Zhu et al. performed a kinetic analysis of IAV-infected porcine macrophages [20]. Both studies found marked changes in cytoskeletal proteins upon IAV infection. We elucidated this further and showed that actin undergoes fragmentation, and these fragments translocate into mitochondria upon IAV infection [19]. Our results suggest that actin fragments, fractins, are involved in the disruption of mitochondrial membranes during apoptosis of virusinfected cells. Actin fragmentation was associated with mitochondrial translocation of RIG-I/MAVS signaling components, which are involved in viral genome recognition and activation of antiviral cytokine production. We also showed that intact actin cytoskeleton structure is crucial for proper activation of antiviral response. Later we made a similar observation in human keratinocytes which were transfected with poly $(\mathrm{I}: \mathrm{C})$ mimicking double-stranded RNA that is formed during viral replication [21], and showed that cytokeratin network is disrupted in a caspase-dependent manner upon dsRNA stimulation as well as upon Encephalomyocarditis virus or Vesicular stomatitis virus infection. These studies also nicely illustrate the power of 2-DE analysis to detect protein fragmentation events.

HIV infection in microglia, which act as the main immune cells of the central nervous system, has been studied using DIGE [22-23], a variant of 2-DE where samples are labelled with different fluorescent dyes, mixed, and the proteins are separated in one 2-DE gel. Labelled proteins are visualized with scanning the gel with different excitation wavelengths, and the resulting protein patterns are compared. The main advantage of DIGE is that it avoids the inter-gel variation of standard 2-DE where all the samples are analyzed with individual gels, in addition, the quantification accuracy of DIGE is very good compared to e.g. silverstaining. Wang and co-workers utilized DIGE and co-culture systems of HIV-1-infected mouse astrocytes and microglia to study the effect of HIV-1-infected astrocytes on the biology of microglia [23]. They provided novel information that HIV-1-infected astrocytes increase the neurotoxicity of HIV-1-infected microglia. The findings suggest that the dynamics of permissive and productive HIV-1 infection in microglia is regulated by intercellular interactions with infected astrocytes, and promote HIV-1 related neuroinflammation and neurodegeneration.

\subsection{MS-based proteomics studies of macrophage response to influenza A virus infection}

Several recent reports have used MS-based proteomics to characterize IAV infection in macrophages. Cheung et al. [24] used label-free quantitative proteomics to characterize differences in highly pathogenic H5N1 and seasonal H1N1 influenza virus infection in MDMs and Liu et al. [16] used SILAC to characterize H1N1 IAV infection in human alveolar macrophages. Both these studies were done using total cellular lysates. The work by Cheung et al. [24] showed that highly pathogenic H5N1 strain of IAV induces significant changes in MDM proteome already at $1 \mathrm{~h}$ post-infection, especially in proteins related to translational machinery. Liu et al. identified a total of 1214 proteins from alveolar macrophages, and 875 of those proteins were reliably quantified [16]. The expression of 106 proteins was statistically significantly altered at $24 \mathrm{~h}$ post-infection, and a high up-regulation of interferon inducible proteins was seen [16]. In addition, the expression of proteins involved in inflammatory response, apoptosis, and redox state was altered in response to IAV infection.

To gain spatio-temporal information how IAV manipulates macrophage proteome, we performed a kinetic, quantitative sub-cellular proteome analysis of human MDMs [25]. We analyzed the changes in nuclear, mitochondrial and cytoplasmic proteomes, as well as in the global protein secretion (secretome) upon IAV infection in three timepoints using iTRAQ. We identified almost 3500 distinct proteins and showed that viral infection regulates the expression and/or subcellular localization of more than one thousand host proteins at early phases of infection [25].

NLRP3 inflammasomes are multimeric cytosolic protein complexes of the innate immune system that are activated in human macrophages in response to viral infection. Upon activation NLRP3 inflammasomes induce inflammation and they are essential for the antimicrobial defense. Assembly of NLRP3 inflammasome triggers the activation of inflammatory cysteine protease caspase- 1 and following proteolytic processing and secretion of pro-inflammatory cytokines Interleukin (IL)-1 $\beta$ and IL-18. The function of NLRP3 inflammasome in caspase-1mediated processing of these cytokines during influenza A virus infection was demonstrated almost two decades ago [26]. After this, experiments with NLRP3 deficient mice have confirmed the central role of NLRP3 inflammasome in innate immune response against IAV infection in vivo [27-29]. Our proteomic study [25] demonstrated a rapid cytoplasmic leakage of lysosomal proteases, cathepsins, and the secretion of these enzymes following influenza A virus infection of human macrophages. Following functional studies showed that cathepsin activity is essential for NLRP3 inflammasome activation in response to influenza A virus infection [25]. In another study we used GeLC-MS/MS analysis to characterize the secretome of macrophages that had been activated through cytoplasmic dsRNA-recognition pathways [30]. This showed that cathepsin secretion is activated upon dsRNA, and similarly to virus infection this dsRNA-induced NLRP3 inflammasome activation was dependent on cathepsin activity [30]. These proteomics studies have revealed novel mechanisms how NLRP3 inflammasome is activated in human macrophages during viral infection $[25,30]$. Our sub-cellular proteomics study also showed that Bax is translocated onto the mitochondria and cytochrome $c$ leaks into the cytoplasm in response to influenza A virus infection [25]. These translocations are classical signs of mitochondrial apoptosis, programmed cell death and in line with the results by Liu et al. on macrophage response to H1N1 infection [16]. Similarly to NLRP3 inflammasome activation, apoptosis of virus-infected cells was abrogated in the presence of cathepsin inhibitors [25].

We have also characterized the phosphoproteome of IAV infected human MDMs using phosphopeptide enrichment with IMAC (immobilized metal ion affinity chromatography) combined with LCMS/MS analysis [31]. We identified $>4000$ nonredundant phosphosites and the phosphorylation status of more than one thousand macrophage proteins was changed upon IAV infection. Bioinformatics analysis of the phosphoproteome data indicated that the phosphorylation of proteins involved in the ubiquitin/proteasome pathway and antiviral responses were regulated during IAV infection. Similarly, proteins involved in small GTPase-, mitogen-activated protein kinase-, and cyclin-dependent kinase (CDK) -signaling were regulated by phosphorylation upon IAV infection. In particular, IAV infection had a major influence on the phosphorylation profiles of a large number of CDK substrates. Functional studies with CDK inhibitors prevented IAV replication both in vitro and in vivo [31]. Therefore targeting these kinases with small-molecule inhibitors could be a novel way to treat severe IAV infections. The data 
also suggest a new paradigm: host signaling molecules regulating viral replication can be new targets of antiviral drugs. In addition to analyzing phosphorylation changes in host proteins, also the phosphorylation of influenza virus proteins has been analyzed with proteomics. Hutchinson et al. utilized LC-MS/MS to map the phosphoproteomes of different IAV strains and one influenza B strain [32]. They identified 36 novel phosphorylation sites in influenza proteins. The study showed a key role for phosphorylation in the IAV life cycle. Very recently, also the phosphoproteome of Herpes Simplex Virus-1 (HSV-1) was mapped [33]. Kulej et al. identified 67 viral proteins and quantified 571 phosphorylation events (465 with high confidence site localization) on viral proteins. Together these studies showed that phosphorylation events are abundant on viral proteins, and suggests that inhibition of viral protein phosphorylation could be a novel antiviral strategy.

\subsection{MS-based proteomics to characterize HIV-1 infection in human macrophages}

Several studies have utilized MDMs as cell models to study the interplay between macrophages and HIV-1. Kraft-Terry and co-workers used pulsed stable isotope labeling of amino acids in cell culture (pSILAC) to study alterations in protein synthesis in MDMs following HIV-1 infection [34]. Expression of proteins related to antigen presentation and interferon-induced antiviral response were substantially up-regulated in HIV-1-infected MDMs. In contrast, proteins involved in metabolic and DNA packaging activities were down-regulated in macrophages infected with HIV-1. This study highlights a range of anti-retroviral mechanisms that develop in macrophages to prevent spread of HIV-1. Haverland et al. utilized SWATH-MS to study protein expression in uninfected and HIV-1-infected MDMs [35]. They identified and quantified 3608 proteins of which 420 were differentially expressed upon HIV-1 infection. Bioinformatics analyses showed that especially the expression of transcription regulator proteins was regulated by infection. In addition, dysregulation of RNA processing was seen. Both aforementioned studies indicate that although macrophages can mount substantial anti-retroviral response, HIV-1 can regulate the expression of host proteins that promote virus survival [34-35].

Barrero et al. utilized SILAC to study the effect of HIV-1 protein Vpr on macrophage protein expression [36]. They used monocytic U937 cells which were differentiated into macrophages and these cells were transduced with adenovirus harboring the Vpr gene. Forced expression of Vpr altered the expression of 136 proteins and the data showed altered expression of many proteins related to cell metabolism. The authors conclude that HIV-1 hijacks macrophages via Vpr to express proteins that facilitate viral replication as well as long-term survival of macrophages [36].

\subsection{Proteomics to study macrophage response to porcine virus infection}

Quantitative proteomics has been used to study changes in protein expression levels in pulmonary alveolar macrophages (PAMs) following porcine reproductive and respiratory syndrome virus (PRRSV) and porcine circovirus type 2 (PCV2) infection using both 2-DE and MS-based proteomics [37-40].

Ding et al. used used 2-DE with silver-staining to characterize PRRSV infection 24 hpi [37] and Zhou et al. used DIGE to compare macrophage response to highly pathogenic PRRSV strain and attenuated strain at 48 hpi [38]. Ding et al. identified 29 proteins to be regulated upon infection. These were related to morphogenesis, protein synthesis, metabolism, stress response, the ubiquitin-proteasome pathway and signal transduction [37]. Zhou et al. found that 153 proteins had significant different expression upon expression in DIGE analysis, and were able to identify 24 proteins from the gel. The virulent strain showed upregulated expression of pyruvate kinase $\mathrm{M} 2$, heat shock protein beta-1, and proteasome subunit alpha type 6 suggesting that these proteins are involved in the pathogenic mechanisms of PRRSV [38].
PRRSV and PCV2 infection in macrophages have also been studied utilizing iTRAQ-labeling and LC-MS/MS analysis of total cellular lysates $[39,40]$. Both these kinetic studies identified $>800$ proteins and showed altered expression of approximately 150 proteins upon infection. These studies showed that in response to PRRSV and PCV2 infection differentially expressed proteins are related to biological processes including virus binding, cell structure, signal transduction, and cell adhesion [39, 40].

In addition, label-free quantitative phosphoproteomic analysis has been used to analyze protein phosphorylation changes in PAMs following PRRSV infection [41]. PRRSV infection of PAMs altered the phosphorylation status of over 240 proteins. Interaction analysis of the data revealed that proteins that were regulated by phosphorylation following PRRSV infection are involved in cellular assembly and organization, protein synthesis, molecular transport, and signal transduction. Pathway analysis in turn suggested that inflammatory cytokine production following PRRSV infection is likely due to the activation of mitogenactivated protein kinases and NF- $\mathrm{B}$ signal pathway [41].

\section{Global protein secretion studies of virally infected macrophages}

Protein secretion through multiple pathways is an important part of immune responses. Immune cells secrete for example small soluble proteins, cytokines and chemokines that are needed for proper coordination of host cell response to different pathogens. However, recent system-level characterizations using proteomics methods have shown that immune cells activate a much more global protein secretion than just secretion of cytokines and chemokines [42,43]. We have extensively studied global protein secretion from human macrophages upon different activation stimuli using various proteomics workflows including 2-DE, GeLC-MS/MS, and iTRAQ [25,30,44-50]. From the technical point of view it is important in these analyses to use serum-free growth media and test the cell culture conditions for optimal cell viability. This is critical for obtaining good quality proteome data with minimal or no contamination from serum proteins and to avoid identifying proteins leaking from dying cells. We used iTRAQ to characterize macrophage secretome upon IAV and herpes simplex virus 1 (HSV-1) infection [25, 44 ]. HSV-1 is a common pathogen infecting the majority of people worldwide at some stage in their lives. It is an icosahedral, enveloped, nuclear-replicating, double-stranded DNA virus. Our studies revealed massive release of danger signal proteins and identified secreted host factors that have a role in antiviral defense in response to IAV and HSV-1 infection in MDMs. In addition, these studies also revealed that many interferon-inducible proteins are secreted in response to viral infection $[25,44]$. The results suggest that interferon-inducible proteins have important extracellular antiviral functions.

In addition to viral infection, we have characterized macrophage secretome upon stimulation upon ATP, monosodium urate (MSU), $\beta$ glucans and intracellular lipopolysaccaharide (LPS) stimulation [4548,50]. Extracellular ATP and MSU are endogenous danger signals known to activate inflammatory responses. $\beta$-glucans are the main constituents of fungal cell walls and LPS is the major cell wall component of Gram-negative bacteria. Interestingly, bioinformatic characterization of all the proteins identified from macrophage secretomes upon different stimuli revealed that most of the proteins do not have the signal sequence required for classical ER/Golgi-mediated secretion $[25,44,45$, $47,50]$. Proteins that do not carry an N-terminal signal peptide are released unconventionally. They are packed into various types of membrane-enclosed structures, collectively called "extracellular vesicles" (EVs). Exosomes are small, 30-150 nm membrane vesicles secreted by most cell types in vitro. ExoCarta is a database providing the contents that have been identified in exosomes in multiple organisms [51]. Most of the proteins identified from macrophage secretomes were found in ExoCarta. These proteomics studies indicate that vesicle-mediated protein secretion is the key secretory pathway activated in 
macrophages in response to different pathogens and endogenous danger signals.

Also protein secretion upon HIV infection has been studied using proteomics. Already ten years ago Ciborowski et al. published secretome analysis of HIV-1-infected human MDMs using SDS-PAGE and MS [52]. Differentially secreted proteins included enzymes, cytoskeletal, redox, and immunoregulatory proteins. The key findings of this study were validated by Western blot analysis. Secretome analysis has been performed in macrophages cultured from peripheral blood mononuclear cells isolated from women having HIV-1 associated with neurocognitive disorders [53]. This study identified possible biomarkers for HIV-1 associated with neurocognitive disorders. In addition, AP-MS has been used to generate an interactome map of host cathepsin B -protein from uninfected and HIV-1-infected MDM cell culture supernatants [54]. The novel data presented in the study indicate that cathepsin B and its' interacting proteins secreted by HIV-1-infected macrophages contribute to neuronal apoptosis.

EVs are important for intercellular communication. Most attention so far has focused on tumor-derived EVs. However, there is increasing evidence that EVs from both non-immune and immune cells have important roles in immune regulation. When released from stimulated cells, they have been shown to modulate the immune response. To gain detailed information on EV-mediated protein secretion events triggered by IAV infection we analyzed the proteome changes in EVs released from macrophages upon IAV infection using GeLC-MS/MS [49]. Proteins secreted in response to IAV infection included many proteins involved in translation, such as components of spliceosome machinery and the ribosome. The data also shows that EVs derived from IAV-infected macrophages contain antiviral cytokines, fatty acid-binding proteins, copper metabolism Murr-1 domain proteins, and autophagy-related proteins. These results suggest EV-mediated protein secretion has an important role in macrophages antiviral response. In addition to viral infections, we have seen that EV-mediated protein secretion is activated also in response to bacterial and fungal infections [46,50].

Kadiu et al. characterized the proteomes of EVs, including exosomes and microvesicles, that were immunoaffinity purified form uninfected and HIV-infected MDMs [55]. They observed mature HIV-1 by electron microscopy in exosomes, and exosomes contained $7-17 \%$ viral proteins compared to total protein content. The results of this study suggest an essential role for exosomes as facilitators of HIV-1 infection. In general, HIV-1 infection is modulated by exosomes in many ways and exosomes are targeted by HIV-1-derived factors [56].

Viruses use virions to spread between hosts, and virions often contain many host proteins that are required for viral transmission. Hutchinson and co-workers utilized ultracentrifugation through a sucrose gradient and MS to gain detailed quantitative information about the protein composition of virions formed by different influenza viruses [57]. This study showed that conserved virion architecture is maintained across different combinations of virus and host. They also demonstrate that virions have many host proteins that are found in exosomes. The data suggests a new concept for virus spread: IAVs utilize vesicles in their exit from infected cells [57]. Linde and co-workers have utilized iTRAQ to study which host proteins are incorporated into HIV-1 virions in CD4-positive T cells and in MDMs [58]. Many of these host proteins have been shown to be relevant to the HIV lifecycle and therefore may be novel targets for anti-retroviral therapy.

\section{Concluding remarks}

The term 'proteome' was introduced a little over twenty years ago and the possibilities proteomics could offer for biomedical research and drug discovery were realized very soon after that [59-61]. However, this first 'proteomics hype' encountered serious difficulties with proteomics methods being not sensitive and robust enough for routine analysis. After intensive period of development for MS-instruments, data analysis tools, and sample preparation methods proteomics has reached the level where it is possible to identify, quantify and characterize thousands of proteins from limited amount of cellular samples in one experiment. An elegant demonstration of this is a very recent paper by Rieckmann et al. [43] where the authors describe a social network architecture of human immune cells using quantitative proteomics. They characterized 28 human primary hematopoietic cell populations that were sorted by flow cytometry using single-run MSanalysis approach. This resulted in the identification of approx. 7500 proteins per measurement [43].

New developments in proteomics also constantly provide novel possibilities for advanced proteome analysis. An example of an emerging proteomics technique which is likely to find wide-spread use in virology and immunology in general is single-cell proteomics using mass cytometry. It combines the cellular analysis principles of traditional fluorescence-based flow cytometry with the selectivity and quantitative power of inductively coupled plasma-mass spectrometry. With mass cytometry it is possible to multiplex up to 40 independent measurements and gain comprehensive understanding of cell phenotypes, signaling pathways and function on a single cell level $[62,63]$.

Proteomics has already significantly contributed to our understanding of macrophage response to viral infection, and identified several hundreds of host proteins regulated by different viruses. Most of these studies have been focusing on analyzing changes in protein expression levels upon infection. To get more complete view on host response to viral infection, future studies should also focus on analyzing how viral infection regulates host proteins' post-translational modifications (PTMs) on a global scale. There are already some phosphoproteome studies on macrophage response to viral infection $[31,41]$ but no other PTMs have been studied on a global scale. An especially interesting PTM in this respect is ubiquitination which is known to be have crosstalk between protein phosphorylation in innate immunity $[64,65]$. Several studies have shown that protein secretion is an important part of macrophages response to viral infection $[25,44,49]$. Here, future studies are needed to elucidate the role of unconventional protein secretion in antiviral response. For this, the proteome composition of EVs secreted upon viral infection need to be first characterized, and also the possible PTMs in EV proteins. In addition, we and others have shown that EVmediated protein secretion is induced following NLRP3 inflammasome activation $[45,46,48,66]$. In future studies proteomics techniques could be exploited to define the intracellular signaling pathways that are essential for the activation EV-mediated protein secretion induced by virus-activated NLRP3 inflammasome.

In summary proteomics offers unique opportunities for in-depth elucidation of host response to viral infection and host-virus interactions, and the methods have reached the state where proteomics should be routinely applied to virology research. However, this is still not the case for most laboratories focusing their research in virology. To have true integration of proteomics into virology more education is needed for biomedical students and researchers so that they would understand how proteomics could contribute to their research. Also multidisciplinary research having seamless collaboration between virology, proteomics and bioinformatics researchers is needed to fully utilize the possibilities proteomics can offer for fundamental new discoveries in virology.

\section{Transparency document}

The Transparency document associated with this article can be found, in online version.

\section{References}

[1] H. Koppensteiner, R. Brack-Werner, M. Schindler, Macrophages and their relevance in human immunodeficiency virus type I infection, Retrovirology 9 (2012) 82.

[2] X.J. Meng, Emerging and re-emerging swine viruses, Transbound. Emerg. Dis. 59 (Suppl. 1) (2012) 85-102. 
[3] M.P. Weekes, P. Tomasec, E.L. Huttlin, C.A. Fielding, D. Nusinow, R.J. Stanton, E.C. Wang, R. Aicheler, I. Murrell, G.W. Wilkinson, P.J. Lehner, S.P. Gygi, Quantitative temporal viromics: an approach to investigate host-pathogen interaction, Cell 157 (2014) 1460-1472.

[4] X. Luo, K.A. Carlson, V. Wojna, R. Mayo, T.M. Biskup, J. Stoner, J. Anderson, H.E. Gendelman, L.M. Meléndez, Macrophage proteomic fingerprinting predicts HIV-1associated cognitive impairment, Neurology 60 (2003) 1931-1937.

[5] K.A. Carlson, P. Ciborowski, C.N. Schellpeper, T.M. Biskup, R.F. Shen, X. Luo, C.] Destache, et al., Proteomic fingerprinting of HIV-1-infected human monocyte-derived macrophages: a preliminary report, J. Neuroimmunol. 147 (2004) 35-42.

[6] V. Wojna, K.A. Carlson, X. Luo, R. Mayo, L.M. Meléndez, E. Kraiselburd, H.E. Gendelman, Proteomic fingerprinting of human immunodeficiency virus type 1-associated dementia from patient monocyte-derived macrophages: a case study, J. Neuro-Oncol. 10 (Suppl. 1) (2004) 74-81.

[7] I. Kadiu, M. Ricardo-Dukelow, P. Ciborowski, H.E. Gendelman, Cytoskeletal protein transformation in HIV-1-infected macrophage giant cells, J. Immunol. 178 (2007) 6404-6415

[8] C. Luciano-Montalvo, P. Ciborowski, F. Duan, H.E. Gendelman, L.M. Melendez, Proteomic analyses associate cystatin B with restricted HIV-1 replication in placental macrophages, Placenta 29 (2008) 1016-1023.

[9] K. Garcia, V. Garcia, J. Perez Laspiur, F. Duan, L.M. Melendez, Characterization of the placental macrophage secretome: implications for antiviral activity, Placenta 30 (2009) 149-155.

[10] D.M. Toro-Nieves, Y. Rodriguez, M. Plaud, P. Ciborowski, F. Duan, J. Perez Laspiur, V. Wojna, et al., Proteomic analyses of monocyte-derived macrophages infected with human immunodeficiency virus type 1 primary isolates from Hispanic women with and without cognitive impairment, J. Neuro-Oncol. 15 (2009) 36-50.

[11] M.R. Donnelly, P. Ciborowski, Proteomics, biomarkers, and HIV-1: a current perspective, Proteomics Clin. Appl. 10 (2016) 110-125.

[12] K.K. Lum, I.M. Cristea, Proteomic approaches to uncovering virus-host protein interactions during the progression of viral infection, Expert Rev. Proteomics 13 (2016) 325-340.

[13] N.S. Heaton, N. Moshkina, R. Fenouil, T.J. Gardner, S. Aguirre, P.S. Shah, N. Zhao, L. Manganaro, J.F. Hultquist, J. Noel, D. Sachs, J. Hamilton, P.E. Leon, A. Chawdury, S. Tripathi, C. Melegari, L. Campisi, R. Hai, G. Metreveli, A.V. Gamarnik, A. GarcíaSastre, B. Greenbaum, V. Simon, A. Fernandez-Sesma, N.J. Krogan, L.C. Mulder, H. van Bakel, D. Tortorella, J. Taunton, P. Palese, I. Marazzi, Targeting viral Proteostasis limits influenza virus, HIV, and dengue virus infection, Immunity 44 (2016) 46-58.

[14] M.H. Sieweke, J.E. Allen, Beyond stem cells: self-renewal of differentiated macrophages, Science 342 (2013) 1242974, http://dx.doi.org/10.1126/science.1242974.

[15] K.S. Akagawa, I. Komuro, H. Kanazawa, T. Yamazaki, K. Mochida, F. Kishi, Functional heterogeneity of colony-stimulating factor-induced human monocyte-derived macrophages, Respirology 11 (Jan 2006) (Suppl:S32-6).

[16] L. Liu, J. Zhou, Y. Wang, R.J. Mason, C.J. Funk, Y. Du, Proteome alterations in primary human alveolar macrophages in response to influenza a virus infection, J. Proteome Res. 11 (2012) 4091-4101.

[17] Z. Qin, The use of THP-1 cells as a model for mimicking the function and regulation of monocytes and macrophages in the vasculature, Atherosclerosis 221 (2012) 2-11.

[18] R. Ganji, S. Dhali, A. Rizvi, S. Rapole, S. Banerjee, Understanding HIV-mycobacteria synergism through comparative proteomics of intra-phagosomal mycobacteria during mono- and HIV co-infection, Sci Rep 6 (2016) 22060, http://dx.doi.org/10.1038/ srep22060.

[19] T. Ohman, J. Rintahaka, N. Kalkkinen, S. Matikainen, T.A. Nyman, Actin and RIG-I/ MAVS signaling components translocate to mitochondria upon influenza a virus infection of human primary macrophages, J. Immunol. 182 (2009) 5682-5692.

[20] J. Zhu, W. Zou, G. Jia, H. Zhou, Y. Hu, M. Peng, H. Chen, M. Jin, Analysis of cellular proteome alterations in porcine alveolar macrophages infected with 2009 (H1N1) and classical swine H1N1 influenza viruses, J. Proteome 75 (2012) 1732-1741.

[21] T. Ohman, N. Lietzen, E. Valimaki, J. Melchjorsen, S. Matikainen, T.A. Nyman, Cytosolic RNA recognition pathway activates 14-3-3 protein mediated signaling and caspase-dependent disruption of cytokeratin network in human keratinocytes, J. Proteome Res. 9 (2010) 1549-1564.

[22] T. Wang, N. Gong, J. Liu, I. Kadiu, S.D. Kraft-Terry, R.L. Mosley, D.J. Volsky, P. Ciborowski, H.E. Gendelman, Proteomic modeling for HIV-1 infected microglia-astrocyte crosstalk, PLoS One 3 (2008), e2507.

[23] T. Wang, N. Gong, J. Liu, I. Kadiu, S.D. Kraft-Terry, J.D. Schlautman, P. Ciborowski, et al., HIV-1-infected astrocytes and the microglial proteome, J. NeuroImmune Pharmacol. 3 (2008) 173-186.

[24] C.Y. Cheung, E.Y. Chan, A. Krasnoselsky, D. Purdy, A.T. Navare, J.T. Bryan, C.K. Leung et al., H5N1 virus causes significant perturbations in host proteome very early in influenza virus-infected primary human monocyte-derived macrophages, J Infect Dis 206 (2012) 640-645.

[25] N. Lietzen, T. Ohman, J. Rintahaka, I. Julkunen, T. Aittokallio, S. Matikainen, T.A Nyman, Quantitative subcellular proteome and secretome profiling of influenza a virus-infected human primary macrophages, PLoS Pathog. 7 (2011), e1001340.

[26] J. Pirhonen, T. Sareneva, M. Kurimoto, I. Julkunen, S. Matikainen, Virus infection activates IL- 1 beta and IL-18 production in human macrophages by a caspase-1-dependent pathway, J. Immunol. 162 (1999) 7322-7329.

[27] T. Ichinohe, H.K. Lee, Y. Ogura, R. Flavell, A. Iwasaki, Inflammasome recognition of influenza virus is essential for adaptive immune responses, J. Exp. Med. 206 (2009) $79-87$.

[28] I.C. Allen, M.A. Scull, C.B. Moore, E.K. Holl, E. McElvania-TeKippe, D.J. Taxman, E.H Guthrie, R.J. Pickles, J.P. Ting, The NLRP3 inflammasome mediates in vivo innate immunity to influenza a virus through recognition of viral RNA, Immunity 30 (2009) 556-565.

[29] P.G. Thomas, P. Dash, J.R. Aldridge Jr., A.H. Ellebedy, C. Reynolds, A.J. Funk, W.J Martin, M. Lamkanfi, R.J. Webby, K.L. Boyd, P.C. Doherty, T.D. Kanneganti, The intracellular sensor NLRP3 mediates key innate and healing responses to influenza a virus via the regulation of caspase-1, Immunity 30 (2009) 566-575.

[30] J. Rintahaka, N. Lietzen, T. Ohman, T.A. Nyman, S. Matikainen, Recognition of cytoplasmic RNA results in cathepsin-dependent inflammasome activation and apoptosis in human macrophages, J. Immunol. 186 (2011) 3085-3092.

[31] S. Soderholm, D.E. Kainov, T. Ohman, O.V. Denisova, B. Schepens, E. Kulesskiy, S.Y. Imanishi, et al., Phosphoproteomics to characterize host response during influenza a virus infection of human macrophages, Mol. Cell. Proteomics 15 (2016) 3203-3219.

[32] E.C. Hutchinson, E.M. Denham, B. Thomas, D.C. Trudgian, S.S. Hester, G. Ridlova, A. York, L. Turrell, E. Fodor, Mapping the phosphoproteome of influenza a and B viruses by mass spectrometry, PLoS Pathog. 8 (11) (2012), e1002993.

[33] K. Kulej, D.C. Avgousti, S. Sidoli, C. Herrmann, A.N. Della Fera, E.T. Kim, B.A. Garcia M.D. Weitzman, Time-resolved global and chromatin proteomics during herpes simplex virus type 1 (HSV-1) infection, Mol. Cell. Proteomics 16 (2017) S92-S107 (4 suppl 1).

[34] S.D. Kraft-Terry, I.L. Engebretsen, D.K. Bastola, H.S. Fox, P. Ciborowski, H.E Gendelman, Pulsed stable isotope labeling of amino acids in cell culture uncovers the dynamic interactions between HIV-1 and the monocyte-derived macrophage J. Proteome Res. 10 (2011) 2852-2862.

[35] N.A. Haverland, H.S. Fox, P. Ciborowski, Quantitative proteomics by SWATH-MS reveals altered expression of nucleic acid binding and regulatory proteins in HIV-1-infected macrophages, J. Proteome Res. 13 (2014) 2109-2119.

[36] C.A. Barrero, P.K. Datta, S. Sen, S. Deshmane, S. Amini, K. Khalili, S. Merali, HIV-1 Vpr modulates macrophage metabolic pathways: a SILAC-based quantitative analysis, PLoS One 8 (2013), e68376.

[37] Z. Ding, Z.J. Li, X.D. Zhang, Y.G. Li, C.J. Liu, Y.P. Zhang, Y. Li, Proteomic alteration of Marc-145 cells and PAMs after infection by porcine reproductive and respiratory syndrome virus, Vet. Immunol. Immunopathol. 145 (2012) 206-213.

[38] Y.J. Zhou, J.P. Zhu, T. Zhou, Q. Cheng, L.X. Yu, Y.X. Wang, S. Yang, et al., Identification of differentially expressed proteins in porcine alveolar macrophages infected with virulent/attenuated strains of porcine reproductive and respiratory syndrome virus, PLoS One 9 (2014), e85767.

[39] Q. Lu, J. Bai, L. Zhang, J. Liu, Z. Jiang, J.J. Michal, Q. He, et al., Two-dimensional liquid chromatography-tandem mass spectrometry coupled with isobaric tags for relative and absolute quantification (iTRAO) labeling approach revealed first proteome profiles of pulmonary alveolar macrophages infected with porcine reproductive and respiratory syndrome virus, J. Proteome Res. 11 (2012) 2890-2903.

[40] J. Liu, J. Bai, Q. Lu, L. Zhang, Z. Jiang, J.J. Michal, Q. He, et al., Two-dimensional liquid chromatography-tandem mass spectrometry coupled with isobaric tags for relative and absolute quantification (iTRAQ) labeling approach revealed first proteome profiles of pulmonary alveolar macrophages infected with porcine circovirus type 2 , J. Proteome 79 (2013) 72-86.

[41] R. Luo, L. Fang, H. Jin, D. Wang, K. An, N. Xu, H. Chen, et al., Label-free quantitative phosphoproteomic analysis reveals differentially regulated proteins and pathway in PRRSV-infected pulmonary alveolar macrophages, J. Proteome Res. 13 (2014) $1270-1280$.

[42] F. Meissner, R.A. Scheltema, H.J. Mollenkopf, M. Mann, Direct proteomic quantification of the secretome of activated immune cells, Science 340 (2013) 475-478.

[43] J.C. Rieckmann, R. Geiger, D. Hornburg, T. Wolf, K. Kveler, D. Jarrossay, F. Sallusto, S.S Shen-Orr, A. Lanzavecchia, M. Mann, F. Meissner, Social network architecture of human immune cells unveiled by quantitative proteomics, Nat. Immunol. (Mar 6 2017) (Epub ahead of print)

[44] J.J. Miettinen, S. Matikainen, T.A. Nyman, Global secretome characterization of herpes simplex virus 1-infected human primary macrophages, J. Virol. 86 (2012) 12770-12778.

[45] E. Valimaki, J.J. Miettinen, N. Lietzen, S. Matikainen, T.A. Nyman, Monosodium urate activates Src/Pyk2/PI3 kinase and cathepsin dependent unconventional protein secretion from human primary macrophages, Mol. Cell. Proteomics 12 (2013) 749-763.

[46] W. Cypryk, T. Ohman, E.L. Eskelinen, S. Matikainen, T.A. Nyman, Quantitative proteomics of extracellular vesicles released from human monocyte-derived macrophages upon beta-glucan stimulation, J. Proteome Res. 13 (2014) 2468-2477.

[47] T. Ohman, L. Teirila, A.M. Lahesmaa-Korpinen, W. Cypryk, V. Veckman, S. Saijo, H. Wolff, et al., Dectin-1 pathway activates robust autophagy-dependent unconventional protein secretion in human macrophages, J. Immunol. 192 (2014) 5952-5962.

[48] E. Valimaki, W. Cypryk, J. Virkanen, K. Nurmi, P.M. Turunen, K.K. Eklund, K.E. Akerman, et al., Calpain activity is essential for ATP-driven unconventional vesicle-mediated protein secretion and inflammasome activation in human macrophages, J. Immunol. 197 (2016) 3315-3325.

[49] W. Cypryk, M. Lorey, A. Puustinen, T.A. Nyman, S. Matikainen, Proteomic and Bioinformatic characterization of extracellular vesicles released from human macrophages upon influenza a virus infection, J. Proteome Res. 16 (2017) 217-227.

[50] M.B. Lorey, K. Rossi, K.K. Eklund, T.A. Nyman, S. Matikainen, Global characterization of protein secretion from human macrophages following non-canonical caspase-4/5 inflammasome activation, Mol. Cell. Proteomics (2017).

[51] S. Keerthikumar, D. Chisanga, D. Ariyaratne, H. Al Saffar, S. Anand, K. Zhao, M. Samuel, M. Pathan, M. Jois, N. Chilamkurti, L. Gangoda, S. Mathivanan, ExoCarta: a web-based compendium of exosomal cargo, J. Mol. Biol. 428 (2016) 688-692.

[52] P. Ciborowski, I. Kadiu, W. Rozek, L. Smith, K. Bernhardt, M. Fladseth, M. RicardoDukelow, H.E. Gendelman, Investigating the human immunodeficiency virus type 
1-infected monocyte-derived macrophage secretome, Virology 363 (2007) 198-209.

[53] K. Colon, J. Perez-Laspiur, R. Quiles, Y. Rodriguez, V. Wojna, S.A. Shaffer, J. Leszyk, R.L. Skolasky Jr., L.M. Melendez, Macrophage secretome from women with HIV-associated neurocognitive disorders, Proteomics Clin. Appl. 10 (2016) 136-143.

[54] Y.M. Cantres-Rosario, N. Hernandez, K. Negron, J. Perez-Laspiur, J. Leszyk, S.A. Shaffer, L.M. Melendez, Interacting partners of macrophage-secreted cathepsin B contribute to HIV-induced neuronal apoptosis, AIDS 29 (2015) 2081-2092.

[55] I. Kadiu, P. Narayanasamy, P.K. Dash, W. Zhang, H.E. Gendelman, Biochemical and biologic characterization of exosomes and microvesicles as facilitators of HIV-1 infection in macrophages, J. Immunol. 189 (2012) 744-754.

[56] M.N. Madison, C.M. Okeoma, Exosomes: implications in HIV-1 pathogenesis, Viruses 7 (2015) 4093-4118.

[57] E.C. Hutchinson, P.D. Charles, S.S. Hester, B. Thomas, D. Trudgian, M. MartínezAlonso, E. Fodor, Conserved and host-specific features of influenza virion architecture, Nat. Commun. 5 (2014) 4816.

[58] M.E. Linde, D.R. Colquhoun, C. Ubaida Mohien, T. Kole, V. Aquino, R. Cotter, N Edwards, J.E. Hildreth, D.R. Graham, The conserved set of host proteins incorporated into HIV-1 virions suggests a common egress pathway in multiple cell types, J. Proteome Res. 12 (2013) 2045-2054.

[59] P. Kahn, From genome to proteome: looking at a cell's proteins, Science (1995) 270.369-270.370.

[60] M.R. Wilkins, J.C. Sanchez, A.A. Gooley, R.D. Appel, I. Humphery-Smith, D.F. Hochstrasser, K.L. Williams, Progress with proteome projects: why all proteins expressed by a genome should be identified and how to do it, Biotechnol. Genet. Eng. Rev. 13 (1996) 19-50.

[61] C. O'Brien, Protein fingerprints, proteome projects and implications for drug discovery, Mol. Med. Today 2 (1996) 316.

[62] K.R. Atkuri, J.C. Stevens, H. Neubert, Mass cytometry: a highly multiplexed singlecell technology for advancing drug development, Drug Metab. Dispos. 43 (2015) 227-233.

[63] C. Giesen, H.A. Wang, D. Schapiro, N. Zivanovic, A. Jacobs, B. Hattendorf, P.J. Schüffler, D. Grolimund, J.M. Buhmann, S. Brandt, Z. Varga, P.J. Wild, D. Günther, B. Bodenmiller, Highly multiplexed imaging of tumor tissues with subcellular resolution by mass cytometry, Nat. Methods 11 (2014) 417-422.

[64] S.M. Heaton, N.A. Borg, V.M. Dixit, Ubiquitin in the activation and attenuation of innate antiviral immunity, J. Exp. Med. 213 (2016) 1-13.

[65] C. Chiang, M.U. Gack, Post-translational control of intracellular pathogen sensing pathways, Trends Immunol. 38 (2017) 39-52.

[66] Y. Zhang, F. Liu, Y. Yuan, C. Jin, C. Chang, Y. Zhu, X. Zhang, C. Tian, F. He, J. Wang, Inflammasome-derived exosomes activate NF-kB signaling in macrophages, J. Proteome Res. 16 (2017) 170-178.

[67] M. Larance, A.I. Lamond, Multidimensional proteomics for cell biology, Nat. Rev. Mol. Cell Biol. 16 (2015) 269-280.
[68] R. Aebersold, M. Mann, Mass-spectrometric exploration of proteome structure and function, Nature 537 (2016) 347-355.

[69] L. von Stechow, C. Francavilla, J.V. Olsen, Recent findings and technological advances in phosphoproteomics for cells and tissues, Expert Rev. Proteomics 12 (2015) 469-487.

[70] M. Ricardo-Dukelow, I. Kadiu, W. Rozek, J. Schlautman, Y. Persidsky, P. Ciborowski, G.D. Kanmogne, et al., HIV-1 infected monocyte-derived macrophages affect the human brain microvascular endothelial cell proteome: new insights into bloodbrain barrier dysfunction for HIV-1-associated dementia, J. Neuroimmunol. 185 (2007) 37-46.

[71] I. Kadiu, H.E. Gendelman, Macrophage bridging conduit trafficking of HIV-1 through the endoplasmic reticulum and Golgi network, J. Proteome Res. 10 (2011) 3225-3238.

[72] P. Ciborowski, Y. Enose, A. Mack, M. Fladseth, H.E. Gendelman, Diminished matrix metalloproteinase 9 secretion in human immunodeficiency virus-infected mononuclear phagocytes: modulation of innate immunity and implications for neurological disease, J. Neuroimmunol. 157 (2004) 11-16.

[73] J.N. Brown, J.J. Kohler, C.R. Coberley, J.W. Sleasman, M.M. Goodenow, HIV-1 activates macrophages independent of toll-like receptors, PLoS One 3 (2008), e3664.

[74] C. Luciano-Montalvo, L.M. Meléndez, Cystatin B associates with signal transducer and activator of transcription 1 in monocyte-derived and placental macrophages, Placenta 30 (2009) 464-467.

[75] S. Pathak, G.A. De Souza, T. Salte, H.G. Wiker, B. Asjo, HIV induces both a down-regulation of IRAK-4 that impairs TLR signalling and an up-regulation of the antibiotic peptide dermcidin in monocytic cells, Scand. J. Immunol. 70 (2009) 264-276.

[76] X. Huang, D.K. Stone, F. Yu, Y. Zeng, H.E. Gendelman, Functional proteomic analysis for regulatory T cell surveillance of the HIV-1-infected macrophage, J. Proteome Res. 9 (2010) 6759-6773.

[77] R.A. Raposo, B. Thomas, G. Ridlova, W. James, Proteomic-based identification of CD4interacting proteins in human primary macrophages, PLoS One 6 (2011), e18690. .

[78] M. Arainga, D. Guo, J. Wiederin, P. Ciborowski, J. McMillan, H.E. Gendelman, Opposing regulation of endolysosomal pathways by long-acting nanoformulated antiretroviral therapy and HIV-1 in human macrophages, Retrovirology 12 (2015) 5.

[79] C. Du, H.F. Liu, Y.Z. Lin, X.F. Wang, J. Ma, Y.J. Li, X. Wang, et al., Proteomic alteration of equine monocyte-derived macrophages infected with equine infectious anemia virus, Proteomics 15 (2015) 1843-1858.

[80] E. Chertova, O. Chertov, L.V. Coren, J.D. Roser, C.M. Trubey, J.W. Bess Jr., R.C. Sowder 2nd, E. Barsov, B.L. Hood, R.J. Fisher, K. Nagashima, T.P. Conrads, T.D. Veenstra, J.D. Lifson, D.E. Ott, Proteomic and biochemical analysis of purified human immunodeficiency virus type 1 produced from infected monocyte-derived macrophages, J. Virol. 80 (2006) 9039-9052.

[81] S. Santos, Y. Obukhov, S. Nekhai, M. Bukrinsky, S. Iordanskiy, Virus-producing cells determine the host protein profiles of HIV-1 virion cores, Retrovirology 9 (2012) 65. 